Article

\title{
Design and Evaluation of a MEMS Magnetic Field Sensor-Based Respiratory Monitoring and Training System for Radiotherapy
}

\author{
Yoonjin $\mathrm{Oh}^{1,+}{ }^{+}$, Young-Jin Jung ${ }^{2,3,+} \mathbb{D}^{\mathbb{D}}$, Sang Hyoun Choi ${ }^{4}$ and Dong Wook Kim ${ }^{5, *}$ \\ 1 Department of Radiation Oncology, Samsung Medical Center, Sungkyunkwan University School \\ of Medicine, Seoul 06351, Korea; yoonjin.oh@samsung.com \\ 2 Department of Radiological Science, Dongseo University, Busan 47011, Korea; microbme@dongseo.ac.kr \\ 3 Center for Radiological Environment \& Health Science, Dongseo University, Busan 47011, Korea \\ 4 Division of Medical Radiation Equipment, Korea Institute of Radiological and Medical Sciences, Seoul 01812, \\ Korea; shchoi@kirams.re.kr \\ 5 Department of Radiation Oncology, Kyung Hee University Hospital at Gangdong, Seoul 05278, Korea \\ * Correspondence: dwkim@khnmc.or.kr; Tel.: +82-2-440-7399 \\ + These authors contributed equally to this work.
}

Received: 19 July 2018; Accepted: 18 August 2018; Published: 21 August 2018

\begin{abstract}
The patient's respiratory pattern and reproducibility are important factors affecting the accuracy of radiotherapy for lung cancer or liver cancer cases. Therefore, respiration training is required to induce respiration regularity before radiotherapy. However, the need for specialized personnel, space, and time-consuming training represent limitations. To solve these problems, we have developed a respiratory monitoring and training system based on a micro-electro-mechanical-system (MEMS) magnetic sensor. This system consists of a small attaching magnet, a sensor, and a breathing pattern output device. In this study, we evaluated the performance of the signal measurement in the developed system based on the various respiratory cycles, the amplitudes, and the position angles of the magnet and the sensor. The system can provide a more accurate breathing signal graph with lower measurement error and higher spatial resolution than conventional sensor methods by using additional magnet. In addition, it is possible the patient to monitor and train breathing himself by making it easy to carry and use without restriction of time and space.
\end{abstract}

Keywords: radiation therapy; respiratory gating system; MEMS magnetic sensor; respiratory monitoring; respiratory training

\section{Introduction}

Radiation therapy has been developed to enable more accurate treatment by precise three-dimensional control of dose distribution, and leading technologies include three-dimensional conformal radiation therapy (3D-CRT), stereotactic body radiation therapy (SBRT), and intensity-modulated radiation therapy (IMRT) [1-4]. The ultimate goal of radiation therapy is to minimize the side effects that peripheral organs experience while controlling the tumor through the biological effects of radiation by fully radiating the radiation dose to the tumor site and minimizing the radiation dose in the surrounding normal tissue [5-7]. However, radiotherapy for tumors located in the abdominal cavity, such as lung or liver cancer, require careful consideration of the patient's respiration because the patient's free-breathing may cause tumor movement of greater than $2.5 \mathrm{~cm}$ [8-11]. During the CT scan or radiation treatment, changes in tumor location due to the patient's breathing may increase the uncertainty in tumor targeting contouring at the treatment planning stage or target positioning at the treatment stage. Internal target 
volume (ITV), which represents the volume encompassing the clinical target volume (CTV) and the internal motion margin, has been proposed to reduce the uncertainty in radiotherapy that can occur from patient breathing [12-15]. However, an ITV-based treatment plan can damage the surrounding normal organs and lead to complications because of the large treatment area, which includes the extent to which tumor movement is predicted. There is a need for a method to minimize the extent of the treatment range in ITV while still transmitting the appropriate prescription dose to the tumor. Several methods have been developed to meet this need, such as respiration gating radiotherapy that irradiates only a specific phase or amplitude range in periodic respiratory motion, breath-hold radiotherapy that stops breathing during irradiation, and active-breathing control (ABC) that involves a breathing assistance device. However, these methods are not applicable to lung cancer patients who have difficulty with breathing control; in fact, the therapeutic effect may vary according to the stability and reproducibility of the patient's breathing pattern [16-22].

Previous studies have shown that patients with irregular respiratory patterns can change these patterns and develop more regular patterns through respiratory training. Therefore, it is important to offer respiratory training to patients for radiation therapy [23-26]. These findings confirm that the patient's respiratory training prior to treatment can provide more accurate radiation treatment for patients with various breathing patterns. Despite the fact that current clinical practice recommends respiratory training to produce regular breathing before treatment, this therapy has drawbacks, such as the need for support personnel and additional time and space for training.

We have developed a patient respiratory monitoring and training system based on a micro-electro-mechanical-system (MEMS) magnetic field sensor to address the clinical need for a respiratory training system that does not require additional man-power or training space. The MEMS magnetic field sensor measures the intensity of the magnetic field generated by the magnet and also measures the phase of respiratory motion to track the position of the sensor. The respiration training system based on the magnetic field sensor has a higher spatial resolution and lower noise level than the conventional method using the MEMS acceleration sensor, thus providing a high-precision breathing cycle graph to enable accurate respiratory training $[27,28]$. In addition, sensors, interfaces, and systems can be very small, which facilitates ease of use, as patients can carry the training system anywhere without the monitor support. In this study, we evaluated the performance of the developed MEMS magnetic field sensor-based respiratory training system by analyzing the measurement error about various respiratory cycles and amplitudes and the influence about the position change of the magnet and sensor.

\section{Materials and Methods}

\subsection{MEMS-Based Respiratory Monitoring and Training System}

The system consists of hardware and software including an attaching magnet, a sensor, and a display device, as shown in Figure 1a. The magnetic strength of the attaching magnet (emagnet, Seoul, Korea) is 3200 gauss (G) and its dimensions are $25 \times 40 \times 4 \mathrm{~mm}^{3}$. The MEMS-based magnetic field sensor (3-Space ${ }^{\mathrm{TM}}$, Portsmouth, OH, USA) has a size of $35 \times 60 \times 15 \mathrm{~mm}^{3}$, a resolution of 12 bits, a sensitivity of $0.73 \mathrm{mG} /$ digit, and a scale range of $-4.7-4.7 \mathrm{G}$. The sensor is located on the front of the patient's chest, and the magnet is on the patient's back. The sensor detects the strength of the magnetic field. The strength of the magnetic field, i.e., magnetic flux density, is inversely proportional to the distance between the sensor and the magnet, and as the distance increases, the strength of the magnetic field decreases. Therefore, the sensor estimates the position by measuring the intensity of the magnetic field according to the distance between the sensor and the magnet. The magnetic flux intensity signal detected by the sensor changes according to the movement of the chest along with the breath and is displayed on the respiratory pattern displayer through the software. The respiration pattern displaying device has a sampling rate of up to $250 \mathrm{~Hz}$ with a size of $35 \times 60 \times 15 \mathrm{~mm}^{3}$. The software was developed with Python 3.5 (Anaconda Python ver3.5, Austin, TX, USA) in a Windows 10 environment. 


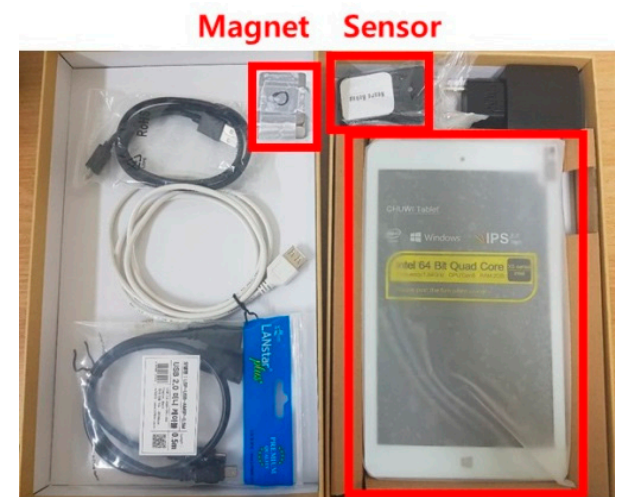

Display device

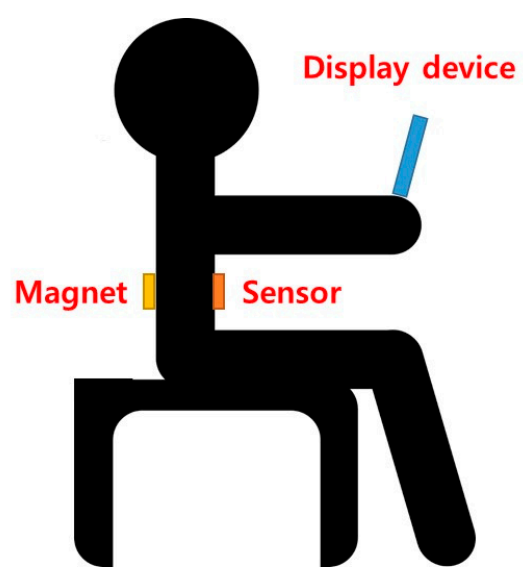

(a)

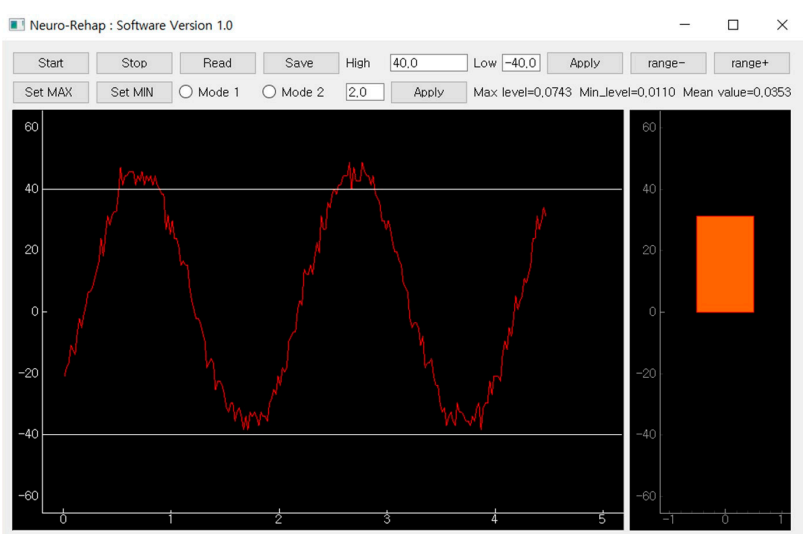

(b)

Figure 1. (a) The hardware part of the respiratory monitoring and training system; (b) breath pattern output device displaying the position of the sensor in a bar graph. In the graph, the $x$-axis represents time(s) and the $y$-axis represents height ratio (\%) of sensor position.

The motion of the sensor attached to the patient's chest is displayed in real time in the form of a bar graph, as shown in Figure 1b, through the respiratory pattern displaying device, so that it enables respiratory training through visual feedback. Figure 2 shows the respiratory monitoring and training process of the system. The Kalman filter is an optimal estimation filter that estimates and outputs the state variables from the measured values using a system model composed of state equations and measurement equations that represent the motion of the state variables. The signal output is obtained by a time-magnetic field strength sine wave graph along each of tri-axis, and we displayed only $y$-axis signal, which is the axis here the sensor and the magnet are located. For respiratory signal measurements, first set the maximum amplitude and minimum amplitude of the data, and select the mode for training. The training mode consists of two types, Mode 1 induces to breathe within the maximum and minimum amplitude values, and Mode 2 induces to breathe at a constant respiratory cycle. To induce respiration, the system show the patient a graph and give a reminder tone. Related information, such as the patient's breathing pattern and time, are stored in an ASCII file for post-analysis. 


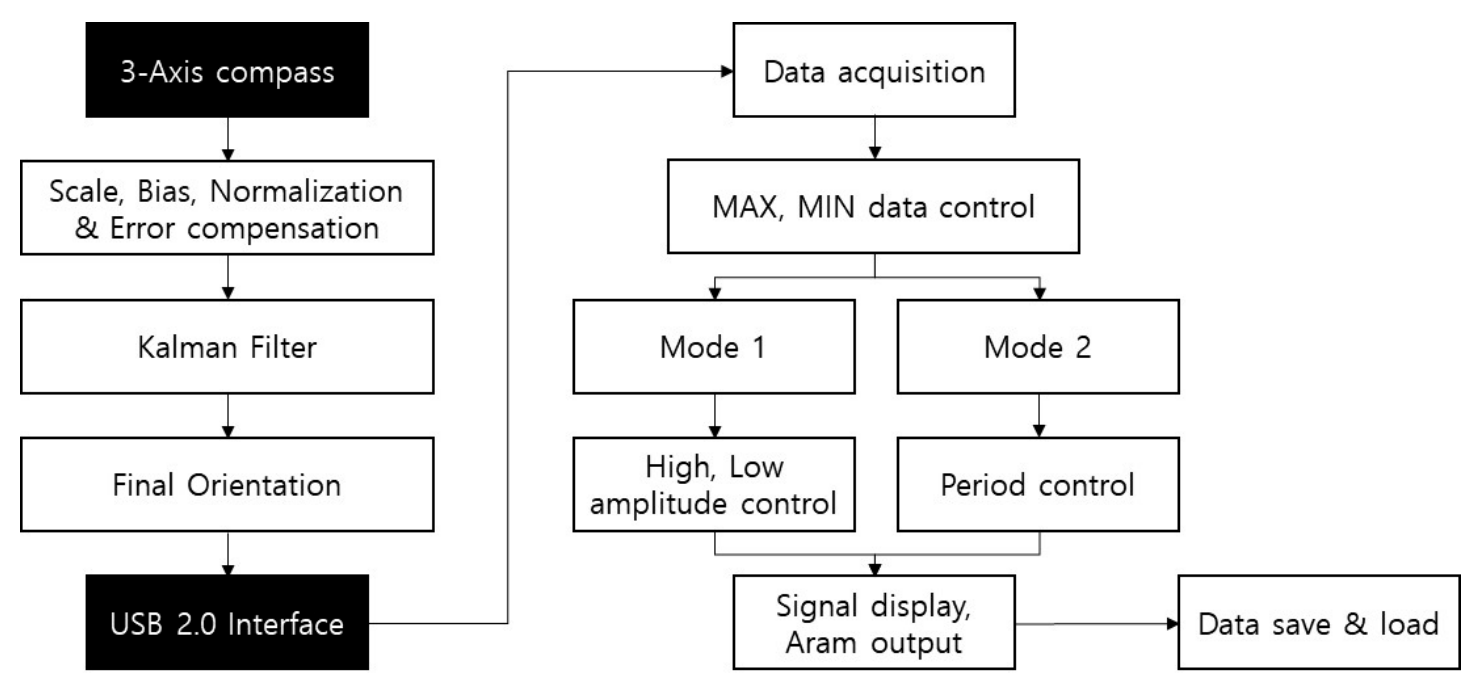

Figure 2. MEMS magnetic field sensor-based respiratory monitoring and training process.

\subsection{Measurement of Respiratory Motion}

The QUASAR ${ }^{\mathrm{TM}}$ Programmable Respiratory Motion Phantom (Modus Medical Devices Inc., London, ON, Canada) was used to simulate various respiratory patterns for the respiratory cycle and amplitude. The QUASAR ${ }^{\mathrm{TM}}$ Phantom consists of an acrylic elliptical cylindrical phantom, drive motor, and cylindrical insert with a 3-cm diameter spherical target. The QUASAR ${ }^{\mathrm{TM}}$ Phantom can adjust the respiratory cycle and amplitude using the respiratory motion program. The amplitude and period of the target motion can be applied up to $3 \mathrm{~cm}$ and $15 \mathrm{~s}$, respectively. The QUASAR ${ }^{\mathrm{TM}}$ Phantom is capable of moving from a minimum of 4 cycles per minute $(\mathrm{cpm})$ to a maximum of $60 \mathrm{cpm}$.

The respiratory cycle of $1,2,3,4$, and $5 \mathrm{~s}$ and the amplitude of 1,2 , and $3 \mathrm{~cm}$ were chosen for measurement in consideration of the average respiratory rate of adults (Figure 3) [29]. The signal measurement time was $0.02 \mathrm{~s}$. The signal acquisition time per case was $60 \mathrm{~s}$ on average and was measured 3 times per case. We used $58 \mathrm{~s}$ of signal data, excluding $2 \mathrm{~s}$, to eliminate noise caused by the initial operation of the QUASAR ${ }^{\mathrm{TM}}$ Phantom. The measurements were normalized to the maximum amplitude of the signal. The period and amplitude error were evaluated based on the actual phantom motion signal data output from the QUASAR ${ }^{\mathrm{TM}}$ Phantom software at the given period and amplitude conditions. The measured values of the system were obtained by calculating the sine function trend equation (Equation (1)); the average period, amplitude, and standard deviation per signal were calculated and compared with the actual phantom signal:

$$
f(x)=A^{*} \sin (B x+C),
$$

A is the amplitude of the measured data, B is the period of the sine function, and $C$ is the phase shift. The period of the measurement data is calculated by dividing by $2 \pi$ phase. The amplitude error and the period error are obtained by subtracting the amplitude and the period of the output signal of the system from the output signal of the actual motion of the QUASARTM Phantom using Equation (1).

\subsection{Position Dependency of Attaching Magnet}

A standard respiratory motion phantom tool (GE Varian 4 D solutions, Varian ${ }^{\circledR}$ Medical Systems, Palo Alto, CA, USA) was used to check the influence of the position change of the magnet on signal measurement. The standard respiratory motion phantom consists of a disk that rotates at a cycle of $5.5 \mathrm{~s}$, a plate that can position the IR reflector, and a drive motor. In this study, we measured the signal by changing the relative angle between the magnet and sensor for $0^{\circ}, 30^{\circ}, 60^{\circ}$, and $90^{\circ}$ at $30 \mathrm{~cm}$ of distance from the sensor, as shown in Figure 4 . The distance of $30 \mathrm{~cm}$ between the sensor and the 
magnet corresponds to the distance during actual breath measurement. The adult waist thickness is in range of 14.6-38.6 cm according to the human body size statistics of Korea. Amplitude error and period error are the difference between the amplitude and period of 30,60, and 90 degrees based on 0 degree where the magnet and the sensor are in line.

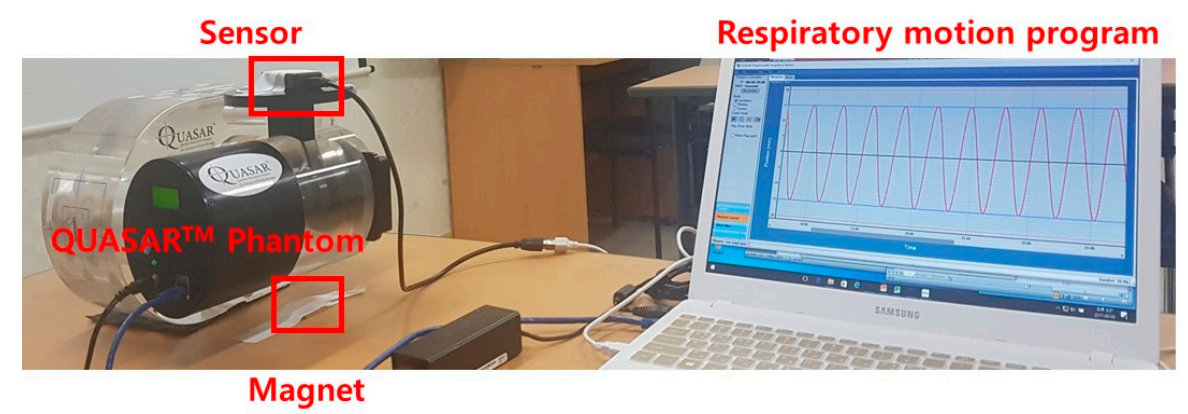

Figure 3. Experimental setup for measuring sensor signals with the QUASAR ${ }^{\mathrm{TM}}$ Programmable Respiratory Motion Phantom and respiratory motion program.

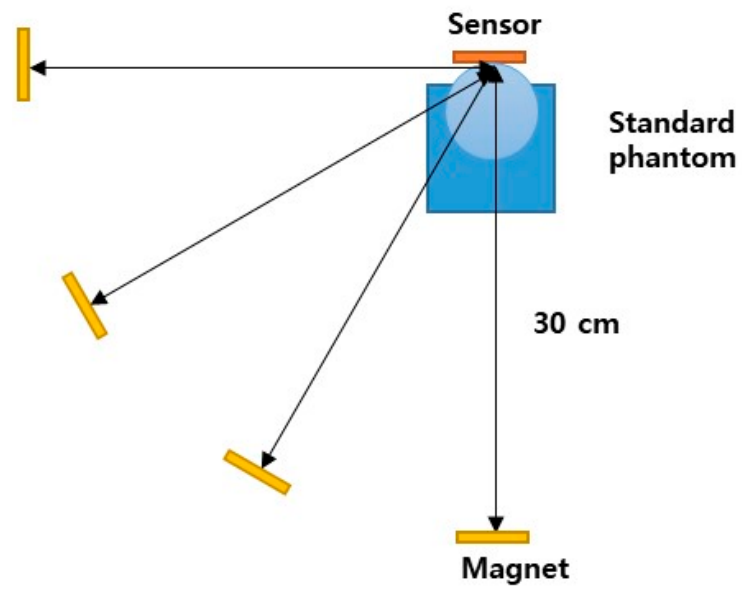

Figure 4. Diagram of the experimental setup for the magnet position test.

\section{Results}

\subsection{Analysis of the Respiratory Signal}

As shown in Figure $5 \mathrm{a}$, the amplitude error of the measured signal was lowest $(22.9 \mu \mathrm{m})$ at $1 \mathrm{~cm}$ amplitude and $5 \mathrm{~s}$ period, and highest $(87.9 \mu \mathrm{m},<0.3 \%)$ at $3 \mathrm{~cm}$ amplitude and $3 \mathrm{~s}$ period. The error of the amplitude for the $1,2,3,4$, and $5 \mathrm{~s}$ periods was 33.0, 33.8, 31.6, 29.4, and $22.9 \mu \mathrm{m}$ for $1 \mathrm{~cm}$ amplitude; 37.7, 57.4, 42.1, 37.3, and $41.0 \mu \mathrm{m}$ for $2 \mathrm{~cm}$ amplitude; and 71.8, 79.5, 87.9, 64.1, and $70.8 \mu \mathrm{m}$ for $3 \mathrm{~cm}$ amplitude, respectively. The amplitude error increased as the set amplitude of the motion phantom became larger.

As shown in Figure $5 b$, the period error of the measured signal was lowest $(0 \mathrm{~ms})$ with a period of 1 or $2 \mathrm{~s}$ and highest $(7.6 \mathrm{~ms},<0.2 \%)$ with a period of $4 \mathrm{~cm}$. The error of the period for the $1,2,3,4$, and 5 s periods was 1.0,3.2, 4.3, 5.1, and $5.3 \mathrm{~ms}$ for $1 \mathrm{~cm}$ amplitude; $0.2,1.9,3.8,3.8$, and $1.3 \mathrm{~ms}$ for $2 \mathrm{~cm}$ amplitude; and 0.0, 0.0,3.3, 7.6, and $2.6 \mathrm{~ms}$ for $3 \mathrm{~cm}$ amplitude, respectively. The error of the respiration period increased as the respiration period increased with amplitude of $1 \mathrm{~cm}$.

As a result of comparing the amplitude of the respiration signal measured according to the respiration period and the amplitude set in the motion phantom, the difference between the expected and measured values showed a tendency to increase as amplitude increased and to decrease slightly as the period increased. 


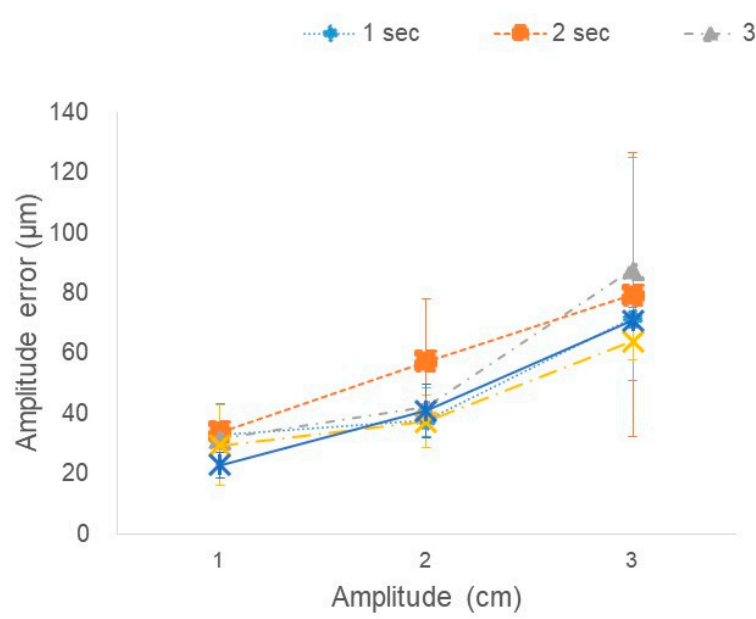

(a)

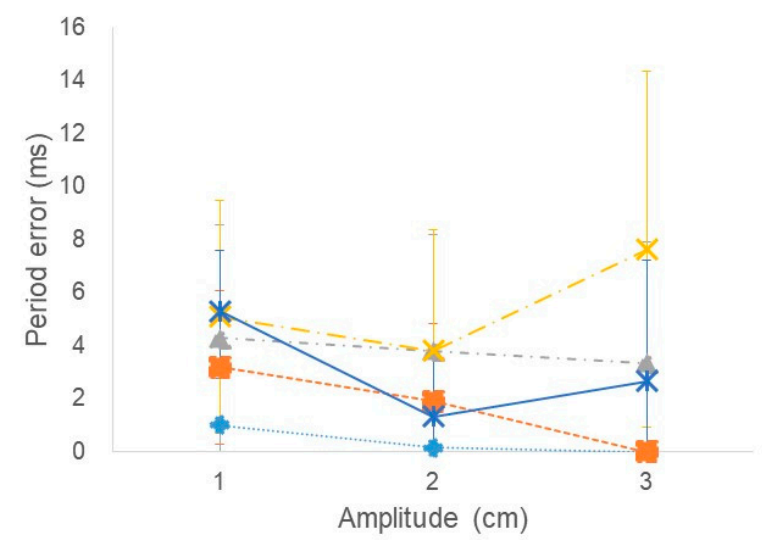

(b)

Figure 5. Error in (a) amplitude $(\mathrm{ms})$ and $(\mathbf{b})$ period $(\mu \mathrm{m})$ by respiration pattern with a period of 1, 2, 3, 4 , and $5 \mathrm{~s}$ and amplitude of 1,2 , and $3 \mathrm{~cm}$.

\subsection{Analysis of Position Dependency of Attaching Magnet}

The error of the measurement signal increased as the magnet angle from the sensor increased. As shown in Figure 6a, the amplitude error of the measurement signal was lowest $(0 \mu \mathrm{m})$ when the magnet was placed in a vertical direction to the sensor and highest $(87.2 \mu \mathrm{m})$ when it was parallel. As shown in Figure $6 \mathrm{~b}$, the period error of the measurement signal was lowest $(0 \mathrm{~ms})$ when the magnet was positioned perpendicular to the sensor. When the angle between the sensor and magnet was $0^{\circ}$, $30^{\circ}, 60^{\circ}$, and $90^{\circ}$, the error of the measured amplitude was $0.0,49.8,77.7$, and $87.2 \mu \mathrm{m}$, and the error of the measured period was $0.0,43.9,14.7$, and $19.6 \mathrm{~ms}$, respectively.

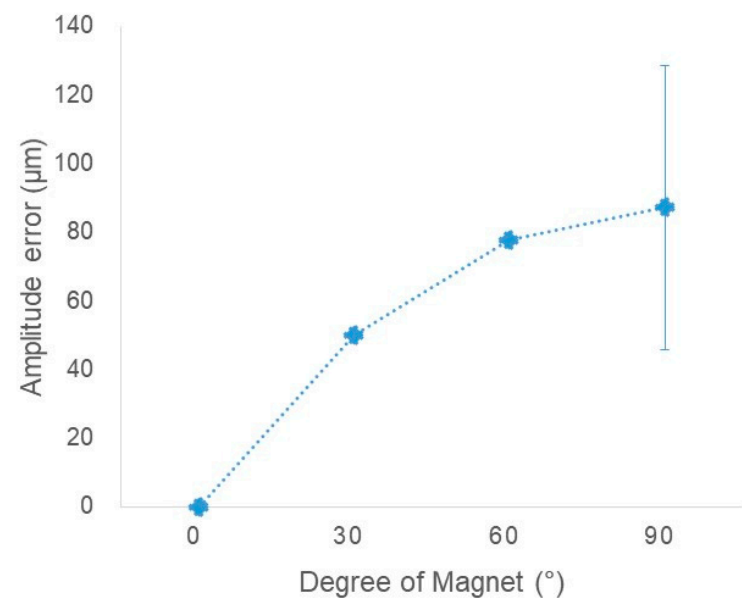

(a)

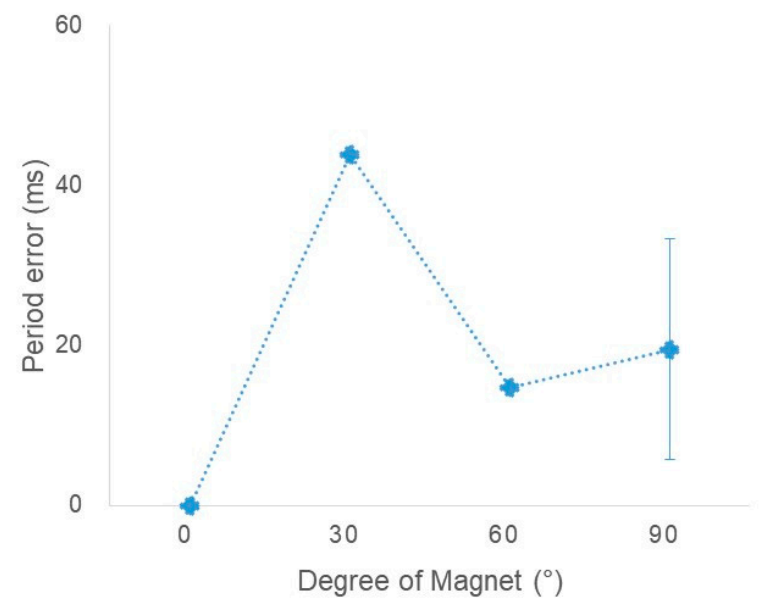

(b)

Figure 6. Error in (a) amplitude (ms) and (b) period $(\mu \mathrm{m})$ by magnet position of $0^{\circ}, 30^{\circ}, 60^{\circ}$, and $90^{\circ}$.

\section{Discussion}

In thoracic or abdominal radiotherapy, the patient's respiration may cause uncertainty of delineation of the target and normal organs and lead to an unnecessary dose increase or decrease of the target and normal organs. In order to monitor such respiratory movements clinically, the techniques of taking a radiographic image and inserting an external marker or surgical node have been introduced. However, radiography and fluoroscopy are relatively costly in time, space, and money and can cause 
additional radiation exposure problems. Marker-based techniques can be affected by the marker attachment position and the patient's posture. Insertion-node-based techniques require invasive methods and increased monitoring preparation time. Despite these drawbacks, adequate respiratory training for the target patient can have positive effects in most cases. In the current study, we developed and evaluated a system that self-monitors respiration without time-space constraints. Because this respiratory monitoring/training system uses magnets with a very high magnetic field, special care is required for patients using electromagnetically sensitive instruments such as pacemakers. Since the intensity of the measuring magnetic field may change according to the user's body thickness, it is necessary to configure/calibrate for each user. To configure system for each user, the user breathes deeply to measure and set the maximum inspiratory and expiratory heights at the beginning.

The system had an error within the range of $0.7 \%$ and $0.2 \%$ for the period and amplitude of respiratory motion. The average period error was $2.9 \mathrm{~ms}$, and the average amplitude error was $49.4 \mu \mathrm{m}$. In 2011, Ono et al. [30] reported the performance of a MEMS angular velocity sensor-based respiration monitoring system. They reported that the maximum difference of the system was $4.3 \%$, the average periodic error was $46.7 \mathrm{~ms}$, and the average amplitude error was $363.3 \mu \mathrm{m}$. These results are similar to reports of the respiration training system based on the MEMS acceleration sensor performed by the present researchers in 2015 [27,31,32]; respiration monitoring based on the MEMS acceleration sensor has a higher error than the MEMS magnetic field sensor. On the other hand, attempts have been made to monitor or train patient respiration with a stereo vision camera. In 2018, Bae et al. evaluated the performance of a stereo vision-based respiratory monitoring system and reported that it had an amplitude error of $2.3 \%$ to $16.3 \%$ and an average amplitude error of $800 \mu \mathrm{m}$. [33]. Using a Kinect v2 camera, Silverstein et al. developed a system for monitoring respiration using information on the surface of the patient without markers and compared their system with existing commercial products, Varian's Real-time Position Management (RPM) System (Varian Medical Systems, Palo Alto, CA, USA) and the Anzai belt system (Anzai Medical Co., Ltd., Tokyo, Japan). They reported that the period measurement error of their system was $77 \mathrm{~ms}$ to $164 \mathrm{~ms}$ [34]. According to the accuracy test of the RPM system used for respiratory monitoring in the present clinical cases, $99.9 \%$ of cases have an error of less than $2 \mathrm{~mm}$ [35]. In 2015, Massaroni et al. evaluated the performance of the opto-electronic plethysmography (OEP) using eight markers and reported that it had a 3D relative motion discrepancy of -64 to $76 \mu \mathrm{m}$ [36]. They reported that the breathing rate error of the fiber Bragg grating (FBG) sensor was -45 to $145 \mathrm{~ms}$ using 42 markers [37]. Our system has similar performance to the results of using these multiple markers and is convenient for use during radiation therapy because only one marker is used. Considering the results of previous studies, the performance of the MEMS magnetic sensor-based respiration training system was comparable or better than other existing systems.

In 2008, Venkat et al. [25] reported that period and amplitude changes can be reduced by about half by appropriate respiratory training. In this way, the MEMS magnetic field sensor-based respiration training system could be used to stabilize unstable breathing patterns by providing a training mode that induces a constant breathing amplitude and cycle. Also, since the system had an average period error of $19.5 \mathrm{~ms}$ and amplitude error of $53.7 \mu \mathrm{m}$ depending on the magnet position, there was not much difference in signal measurement according to the position of the magnet and sensor. This finding indicates that there was little dependence on the user's position.

These results suggest that our system based on the MEMS magnetic field sensor could increase the accuracy of respiration monitoring, as the system has less measurement error for respiratory motion than previous technologies. Moreover, the system is easy to operate and easy to use anytime and anywhere, and it enables patients to monitor and train their own breathing, which is expected to shorten radiotherapy time and improve treatment results. In this study, further review of system responses to long-term use are needed, given that patients require long-term breathing training in general clinical situations. 


\section{Conclusions}

In this study, we evaluated the performance of a respiratory monitoring and training system based on the MEMS magnetic field sensor developed to correct the pattern and reproducibility of respiratory motion in patients and thus increase the accuracy of radiotherapy. The respiration signal period and amplitude error of the system were $0 \mathrm{~ms}$ to $7.6 \mathrm{~ms}$ and $22.9 \mu \mathrm{m}$ to $87.9 \mu \mathrm{m}$, respectively, and there was a maximum difference of $43.9 \mathrm{~ms}$ and $87.2 \mu \mathrm{m}$ depending on the measurement position. These findings indicate that breathing signal measurement was stable and patient breath monitoring was possible in various postures. The system is easy to carry with this performance and can be used by the patients themselves without limitations of time, space and monitoring support. If this system is used for respiration training, it will contribute to the improvement of radiation therapy outcomes by helping to maintain respiration stability and reproducibility during radiotherapy.

Author Contributions: D.W.K. conceived of and designed the experiments; Y.O. performed the experiments; D.W.K. and Y.O. analyzed the data; Y.-J.J. and S.H.C. contributed reagents/materials/analysis tools; Y.O. wrote the paper.

Funding: This research was funded by the General Researcher Program (NRF-2015R1D1A1A09056828) and the Nuclear Safety Research Program (Grant No.1603016) through the Korea Foundation of Nuclear Safety (KOFONS), a granted financial resource from the Nuclear Safety and Security Commission (NSSC), Republic of Korea.

Conflicts of Interest: The authors declare no conflict of interest.

\section{References}

1. Bucci, M.K.; Bevan, A.; Roach, M. Advances in radiation therapy: Conventional to 3D, to IMRT, to 4D, and beyond. Cancer J. Clin. 2005, 55, 117-134. [CrossRef]

2. Palma, D.A.; Verbakel, W.F.; Otto, K.; Senan, S. New developments in arc radiation therapy: A review. Cancer Treat. Rev. 2010, 36, 393-399. [CrossRef] [PubMed]

3. Intensity Modulated Radiation Therapy Collaborative Working Group. Intensity-modulated radiotherapy: Current status and issues of interest. Int. J. Radiat. Oncol. Biol. Phys. 2001, 51, 880-914. [CrossRef]

4. Bhide, S.A.; Nutting, C.M. Recent advances in radiotherapy. BMC Med. 2010, 8, 25. [CrossRef] [PubMed]

5. Hockel, M.; Vaupel, P. Tumor hypoxia: Definitions and current clinical, biologic, and molecular aspects. J. Natl. Cancer Inst. 2001, 93, 266-276. [CrossRef] [PubMed]

6. Tannock, I.F.; Hill, R.P. The Basic Science of Oncology, 2nd ed.; McGraw-Hill, Inc.: New York, NY, USA, 1992; pp. 259-275, ISBN 978-0-071-05316-7.

7. Fowler, J.F. The linear-quadratic formula and progress in fractionated radiotherapy. Br. J. Radiol. 1989, 62, 679-694. [CrossRef] [PubMed]

8. $\quad$ Keall, P.J.; Mageras, G.S.; Balter, J.M.; Emery, R.S.; Forster, K.M.; Jiang, S.B.; Ramsey, C.R. The management of respiratory motion in radiation oncology report of AAPM Task Group 76. Med. Phys. 2006, 33, 3874-3900. [CrossRef] [PubMed]

9. Britton, K.R.; Starkschall, G.; Tucker, S.L.; Pan, T.; Nelson, C.; Chang, J.Y.; Komaki, R. Assessment of gross tumor volume regression and motion changes during radiotherapy for non-small-cell lung cancer as measured by four-dimensional computed tomography. Int. J. Radiat. Oncol. Biol. Phys. 2007, 68, 1036-1046. [CrossRef] [PubMed]

10. Chang, J.; Mageras, G.S.; Yorke, E.; De Arruda, F.; Sillanpaa, J.; Rosenzweig, K.E.; Ling, C.C. Observation of interfractional variations in lung tumor position using respiratory gated and ungated megavoltage cone-beam computed tomography. Int. J. Radiat. Oncol. Biol. Phys. 2007, 67, 1548-1558. [CrossRef] [PubMed]

11. Weiss, E.; Wijesooriya, K.; Dill, S.V.; Keall, P.J. Tumor and normal tissue motion in the thorax during respiration: Analysis of volumetric and positional variations using 4D CT. Int. J. Radiat. Oncol. Biol. Phys. 2007, 67, 296-307. [CrossRef] [PubMed]

12. Ekberg, L.; Holmberg, O.; Wittgren, L.; Bjelkengren, G.; Landberg, T. What margins should be added to the clinical target volume in radiotherapy treatment planning for lung cancer? Radiother. Oncol. 1998, 48, 71-77. [CrossRef]

13. Brady, L.W.; Yaeger, T.E.; Reiff, J.; Class, R.; Mose, S. Encyclopedia of Radiation Oncology, 1st ed.; Springer: Berlin/Heidelberg, Germany, 2013; pp. 585-693, ISBN 978-3-540-85513-2. 
14. Xi, M.; Liu, M.Z.; Deng, X.W.; Zhang, L.; Huang, X.Y.; Liu, H.; Cui, N.J. Defining internal target volume (ITV) for hepatocellular carcinoma using four-dimensional CT. Radiother. Oncol. 2007, 84, 272-278. [CrossRef] [PubMed]

15. Liu, H.H.; Balter, P.; Tutt, T.; Choi, B.; Zhang, J.; Wang, C.; Starkschall, G. Assessing respiration-induced tumor motion and internal target volume using four-dimensional computed tomography for radiotherapy of lung cancer. Int. J. Radiat. Oncol. Biol. Phys. 2007, 68, 531-540. [CrossRef] [PubMed]

16. Mah, D.; Hanley, J.; Rosenzweig, K.E.; Yorke, E.; Braban, L.; Ling, C.C.; Mageras, G. Technical aspects of the deep inspiration breath-hold technique in the treatment of thoracic cancer. Int. J. Radiat. Oncol. Biol. Phys. 2000, 48, 1175-1185. [CrossRef]

17. Berson, A.M.; Emery, R.; Rodriguez, L.; Richards, G.M.; Ng, T.; Sanghavi, S.; Barsa, J. Clinical experience using respiratory gated radiation therapy: Comparison of free-breathing and breath-hold techniques. Int. J. Radiat. Oncol. Biol. Phys. 2004, 60, 419-426. [CrossRef] [PubMed]

18. Liu, H.H.; Koch, N.; Starkschall, G.; Jacobson, M.; Forster, K.; Liao, Z.; Stevens, C.W. Evaluation of internal lung motion for respiratory-gated radiotherapy using MRI: Part II-Margin reduction of internal target volume. Int. J. Radiat. Oncol. Biol. Phys. 2004, 60, 1473-1483. [CrossRef] [PubMed]

19. Jin, J.Y.; Ajlouni, M.; Chen, Q.; Yin, F.F.; Movsas, B. A technique of using gated-CT images to determine internal target volume (ITV) for fractionated stereotactic lung radiotherapy. Radiother. Oncol. 2006, 78, 177-184. [CrossRef] [PubMed]

20. Wong, J.W.; Sharpe, M.B.; Jaffray, D.A.; Kini, V.R.; Robertson, J.M.; Stromberg, J.S.; Martinez, A.A. The use of active breathing control (ABC) to reduce margin for breathing motion. Int. J. Radiat. Oncol. Biol. Phys. 1999, 44, 911-919. [CrossRef]

21. Bloemen-van Gurp, E.; van der Meer, S.; Hendry, J.; Buijsen, J.; Visser, P.; Fontanarosa, D.; Verhaegen, F. Active breathing control in combination with ultrasound imaging: A feasibility study of image guidance in stereotactic body radiation therapy of liver lesions. Int. J. Radiat. Oncol. Biol. Phys. 2013, 85, 1096-1102. [CrossRef] [PubMed]

22. Diaconu, C.; Stephans, K.; Djemil, T.; Videtic, G.; Greskovich, J.; Xia, P. Active Breathing Control Provides an Accurate Method for Stereotactic Body Radiation Therapy of Lung and Liver Tumors. Int. J. Radiat. Oncol. Biol. Phys. 2012, 84, S830. [CrossRef]

23. George, R.; Chung, T.D.; Vedam, S.S.; Ramakrishnan, V.; Mohan, R.; Weiss, E.; Keall, P.J. Audio-visual biofeedback for respiratory-gated radiotherapy: Impact of audio instruction and audio-visual biofeedback on respiratory-gated radiotherapy. Int. J. Radiat. Oncol. Biol. Phys. 2006, 65, 924-933. [CrossRef] [PubMed]

24. Neicu, T.; Berbeco, R.; Wolfgang, J.; Jiang, S.B. Synchronized moving aperture radiation therapy (SMART): Improvement of breathing pattern reproducibility using respiratory coaching. Phys. Med. Biol. 2006, 51, 617-636. [CrossRef] [PubMed]

25. Venkat, R.B.; Sawant, A.; Suh, Y.; George, R.; Keall, P.J. Development and preliminary evaluation of a prototype audiovisual biofeedback device incorporating a patient-specific guiding waveform. Phys. Med. Biol. 2008, 53, N197-N208. [CrossRef] [PubMed]

26. Kini, V.R.; Vedam, S.S.; Keall, P.J.; Patil, S.; Chen, C.; Mohan, R. Patient training in respiratory-gated radiotherapy. Med. Dosim. 2003, 28,7-11. [CrossRef]

27. Sung, J.W.; Yoon, M.G.; Chung, W.K.; Kim, D.W.; Shin, D.O. Study of the Respiratory Monitoring System by Using the MEMS Acceleration Sensor. Prog. Med. Phys. 2013, 24, 61-67. [CrossRef]

28. Hwang, S.B.; Park, M.K.; Park, S.W.; Cho, Y.R.; Lee, D.H.; Jung, H.J.; Ji, Y.H.; Kwon, S.I. Development and Utility Evaluation of Portable Respiration Training Device for Image-guided Stereotactic Body Radiation Therapy (SBRT). Prog. Med. Phys. 2014, 25, 264-270. [CrossRef]

29. Barrett, K.E.; Barman, S.M.; Boitano, S.; Brooks, H. Ganong's Review of Medical Physiology, 23rd ed.; McGraw-Hill Medical: New York, NY, USA, 2009.

30. Ono, T.; Takegawa, H.; Ageishi, T.; Takashina, M.; Numasaki, H.; Matsumoto, M.; Teshima, T. Respiratory monitoring with an acceleration sensor. Phys. Med. Biol. 2011, 56, 6279-6289. [CrossRef] [PubMed]

31. Sung, J.; Yoon, M.; Do Huh, H.; Shin, D.O.; Chung, W.K.; Kim, D.W. Examination of a micro-electro-mechanical system based on a portable respiratory monitoring system. J. Korean Phys. Soc. 2015, 67, 752-756. [CrossRef] 
32. Moon, S.Y.; Sung, J.; Yoon, M.; Chung, M.; Chung, W.K.; Kim, D.W. Evaluation of performance of portable respiratory monitoring system based on micro-electro-mechanical-system for respiratory gated radiotherapy. In Proceedings of the SPIE 9550, San Diego, CA, USA, 9-13 August 2015.

33. Bae, M.; Lee, S.; Kim, N. Development of a robust and cost-effective 3D respiratory motion monitoring system using the kinect device: Accuracy comparison with the conventional stereovision navigation system. Comput. Methods Prog. Biomed. 2018, 160, 25-32. [CrossRef] [PubMed]

34. Silverstein, E.; Snyder, M. Comparative analysis of respiratory motion tracking using Microsoft Kinect v2 sensor. J. Appl. Clin. Med. Phys. 2018, 19, 193-204. [CrossRef] [PubMed]

35. Kim, J.S.; Shin, E.; Shin, J.S.; Ju, S.G.; Han, Y.; Park, H.C.; Choi, D.H. The clinical implementation of 2D dose distribution QA system for the patient specific respiratory-gated radiotherapy. Korean J. Med. Phys. 2010, 21, 127-136.

36. Massaroni, C.; Schena, E.; Saccomandi, P.; Morrone, M.; Sterzi, S.; Silvestri, S. Evaluation of optoelectronic plethysmography accuracy and precision in recording displacements during quiet breathing simulation. In Proceedings of the 2015 37th Annual International Conference of the Engineering in Medicine and Biology Society (EMBC), Milano, Italy, 25-29 August 2015; pp. 1291-1294.

37. Massaroni, C.; Saccomandi, P.; Formica, D.; Presti, D.L.; Caponero, M.A.; Di Tomaso, G.; Schena, E. Design and feasibility assessment of a magnetic resonance-compatible smart textile based on fiber Bragg grating sensors for respiratory monitoring. IEEE Sens. J. 2016, 16, 8103-8110. [CrossRef]

(C) 2018 by the authors. Licensee MDPI, Basel, Switzerland. This article is an open access article distributed under the terms and conditions of the Creative Commons Attribution (CC BY) license (http://creativecommons.org/licenses/by/4.0/). 